\section{Andreas Wirz-Ridolfi}

\title{
Öffentliche Vorträge zur Emeritierung von Dr. med. Brigitte Ausfeld-Hafter, Dozentin für Akupunktur/TCM an der Kollegialen Instanz für Komplementärmedizin KIKOM
}

Der Hörsaal A 003 der Universität Bern konnte am 19. Januar 2012 kaum alle Interessierten fassen, die gekommen waren, um den fünf Vorträgen zu lauschen, die zur Emeritierung von Brigitte Ausfeld-Hafter (Abb. 1) gehalten wurden.

Frau Ausfeld-Hafter war - und ist bis zu ihrem Übertritt in den Ruhestand am 31. Juli 2012 - während 18 Jahren die einzige Universitätsdozentin für Akupunktur und Traditionelle Chinesische Medizin/TCM. Damit war sie weltweit «eine aussergewöhnliche Pionierin unter Pionieren», wie es ein Laudator ausdrückte. Ihre Position als Vertreterin der Komplementärmedizin in der männlich dominierten Welt der Schulmedizin war alles andere als einfach. Durch ihr kritisches und kompromissloses Bestehen auf streng wissenschaftlichen Kriterien hat sie jedoch ihrem Fachgebiet zweifellos zu vermehrter Anerkennung verholfen. Spannend ist auch die Vorgeschichte, die einer der Referenten als Zangengeburt bezeichnet hat. Sie kann als eigentliches Lehrstück schweizerischer Demokratie betrachtet werden. Im Jahr 1992 wurde im Kanton Bern eine Volksinitiative eingereicht, die eine Abteilung für Komplementärmedizin mit Lehrstuhl an der Universität Bern forderte. Da die Regierung jedoch eine Volksabstimmung zu diesem Thema vermeiden wollte, beauftragte sie die Medizinische Fakultät, mit dem Initiativkomitee eine Lösung $\mathrm{zu}$ finden, die zum Rückzug der Initiative zu führen hatte. Nach zähen und harten Verhandlungen von 2 Jahren Dauer beschloss der Regierungsrat schliesslich die Schaffung eines Lehrstuhläquivalents, das dann Kollegiale Instanz für Komplementärmedizin (KIKOM) genannt wurde. Die KIKOM wurde 1994 gegründet und konnte schliesslich im Frühsommer 1995 den Betrieb aufnehmen. Damit kamen vier komplementärmedizinische Fachgebiete zu quasi universitären Weihen, nämlich Anthroposophische Medizin, Homöopathie, Neuraltherapie und eben Akupunktur/TCM. Da sich die vier Disziplinen die kargen räumlichen, finanziellen und personellen Ressourcen teilen müssen, pflegte Frau Ausfeld-Hafter jeweils zu sagen, sie sei nur Inhaberin eines Stuhlbeines dieses Lehrstuhls. Durch diese Nähe wurde jedoch sicher auch der interdisziplinäre Dialog gefördert.

Mit viel Fachwissen, Vehemenz und Geschick hat Frau Ausfeld-Hafter den Aufbau und Ausbau der KIKOM vorangetrieben. Schliesslich konnten die Dozenten der KIKOM die Komplementärmedizin ins Curriculum Humanmedizin der Universität Bern integrieren. Durch ihre Präsenz in der Eidgenössischen Leistungs- und Grundsatzkommission (ELGK) und der Eidgenössischen Kommission für Analysen, Mittel und Gegenstände (EAMGK) hat sie die schweizerische Gesundheitspolitik mitgestaltet. Als Dozentin der KIKOM hat sie neben der Lehre und Forschung auch sieben Dissertationen betreut, die unter ihrer

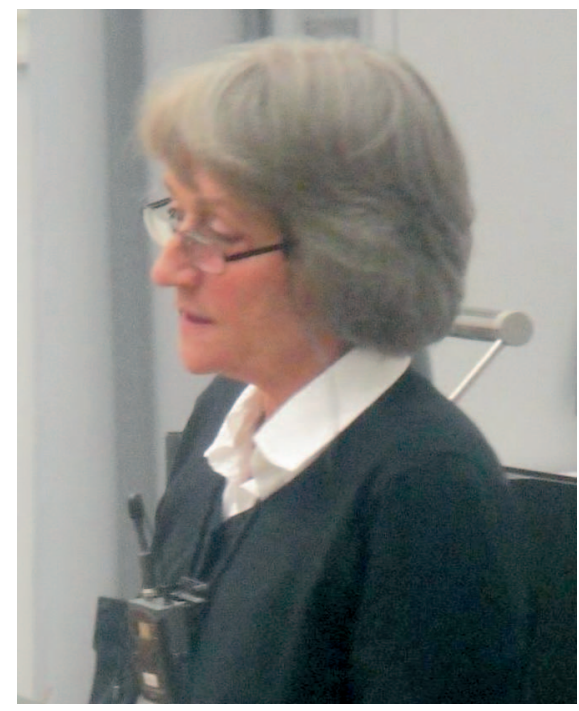

Abb. 1. Rede von Frau Dr. med. Brigitte Ausfeld-Hafter im Rahmen der öffentlichen Vorträge zu ihrer Emeritierung.

Ägide entstanden sind. Ausserdem hat sie sechs Bücher der äusserst lesenswerten Reihe «Komplementäre Medizin im interdisziplinären Diskurs» herausgegeben, erschienen im Verlag Peter Lang, von den kaum zu zählenden Workshops und Vorträgen an nationalen und internationalen Kongressen nicht zu reden. Ein schier unglaubliches Programm, das sie notabene in einer Teilzeitstelle von $25 \%$ bewältigt hatte. Dafür gebührt ihr Dank, Lob und Anerkennung.

Dies geschah auch in den fünf Referaten dieses Abschiedssymposiums, das von Martin Frei-Erb moderiert wurde. Den Anfang machte Adrian Renfer mit seinem langen Referat «Kurze Geschichte der chinesischen

\section{KARGER}

Fax +4976145207 14 Information@Karger.de www.karger.com
(๑) 2012 S. Karger GmbH, Freiburg
Dr. med. Andreas Wirz-Ridolfi

Rebgasse 2, 4153 Reinach, Schweiz

Tel. +44 61-7117478, Fax -7117362

andy.wirz@gmail.com 


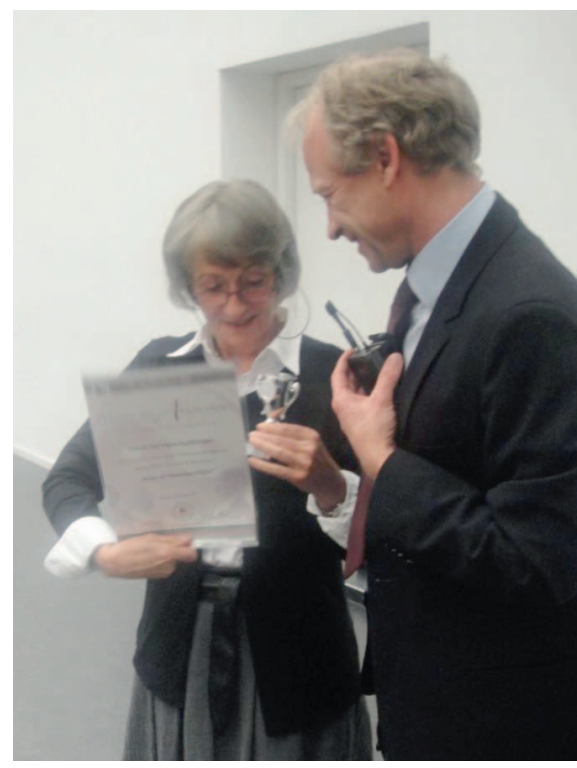

Abb. 2. Ehrung von Frau Dr. med. Brigitte Ausfeld-Hafter durch PD Dr. med. Dominik Irnich.

Philosophie», das in seiner Dichte möglicherweise den einen oder anderen Hörer etwas überforderte. Die vollständigen 38 Seiten können jedoch bei Adrian Renfer bezogen werden. Das nächste Referat hatte den spannendsten Titel aller Vorträge: «Animals are something invented by plants to move seeds around oder zur Komplexizität in holistischen medizinischen Systemen» und wurde gehalten von Ursula Wolf. In ihrem Vortrag entwickelte sie Gedanken zu den verschiedenen Phänomenbereichen des Physischen, Lebendigen, Seelischen und Geistigen, zu deren Emergenz sowie zur prinzipiellen Möglichkeit, deren Gesetzmässigkeiten nach wissenschaftlicher Methode erfassen zu können. Im nächsten Beitrag berichtete Marko Nedeljković über seine schöne Studie «Taiji zur Stressprävention», in der er zeigen konnte, dass regelmässiges Üben von Taiji den Stresshormon-Spiegel tatsächlich zu senken vermag. Er hat dies mit seiner Studiengruppe auch gleich als wohltuende Auflockerung ad oculos demonstriert. Jörg Fritschi zeigte mit informativen Folien den Werdegang des von ihm gegründeten ASA-TCMKongresses, der mittlerweile zum zweitgrössten Akupunkturkongress Europas geworden ist, von 2006 bis 2011 auf.

$\mathrm{Zu}$ guter Letzt beschrieb André Thurneysen in seinem geradezu poetischen Beitrag «Yin-Yang, Dao und Wissenschaft» den Werdegang der KIKOM und der zu verabschiedenden Dozentin. Eingangs führte er ein Zitat von Heinrich von Kleist an, dessen 200. Todestag sich kürzlich jährte: «Man könnte die Menschen in zwei Klassen abtheilen: in solche, die sich auf eine Metapher und solche, die sich auf eine Formel verstehen. Deren, die sich auf beides verstehen, sind $\mathrm{zu}$ wenige, sie machen keine Klasse aus.» Sein Referat schloss er mit der Feststellung, dass Brigitte Ausfeld-Hafter ohne Zweifel der dritten Klasse nach Kleist angehört - nicht ohne anzufügen, dass die Ärzte überhaupt dieser dritten Klasse angehören sollten, wenn sie ihren Beruf patientengerecht ausüben wollen.

Mit einem musikalischen Intermezzo leitete Michael Kofmehl, der seinem Alphorn archaische und trotzdem feinfühlige Melodien entlockte, dann zum anschliessenden Apéro über.

Eine besondere Ehrung erfuhr Brigitte Ausfeld-Hafter durch PD Dr. Dominik Irnich, 2. Vorsitzender und Leiter des Fortbildungszentrums der Deutschen Ärztegesellschaft für Akupunktur e.V., der eigens aus München angereist kam: Sie erhielt für ihre Verdienste um die Akupunktur in Deutschland das Diplom «Meister der Akupunktur», einen Pokal sowie einen Korb voller Zutaten für ihr leibliches Wohl (Abb. 2).

Das letzte Wort hatte Brigitte Ausfeld-Hafter mit einem Zitat, das man sich unbedingt merken muss: «Gesegnet sind diejenigen, die nichts zu sagen haben und dies nicht auch noch ausdrücken wollen.»

Auf wen geht dieser kluge Aphorismus zurück? Der Münchner Komiker Karl Valentin sagte: «Gesegnet seien jene, die nichts zu sagen haben und trotzdem den Mund halten.» Doch eleganter drückte es die englische Schriftstellerin George Eliot (18191880) aus: «Blessed be the man who, having nothing to say, abstains from giving wordy evidence of the fact!»

Aus dem Munde der baldigen Emerita ist dies natürlich ein Ladygleiches Understatement, denn wenn jemand etwas zu sagen hat, dann ist es Brigitte Ausfeld-Hafter!

In diesem Sinne, liebe Brigitte, hoffen wir, dass Du der Wissenschaft trotz verdientem otium cum dignitate noch etwas erhalten bleibst und es nicht heisst: «Aus den Augen - aus dem Feld.» 